\title{
Subcarrier index-power modulated optical OFDM with dual superposition multiplexing for IMDD PON systems
}

Halabi, F.; Chen, L.; Giddings, R.P.; Hamie, A.; Dumas, Y.; Freyssinet, P.; Aupetit-Berthelemot, C.; Tang, J.M.

\section{Optics Communications}

DOI:

10.1016/j.optcom.2018.10.017

Published: 15/02/2019

Peer reviewed version

Cyswllt i'r cyhoeddiad / Link to publication

Dyfyniad o'r fersiwn a gyhoeddwyd / Citation for published version (APA):

Halabi, F., Chen, L., Giddings, R. P., Hamie, A., Dumas, Y., Freyssinet, P., Aupetit-Berthelemot, C., \& Tang, J. M. (2019). Subcarrier index-power modulated optical OFDM with dual superposition multiplexing for IMDD PON systems. Optics Communications, 433, 190-194. https://doi.org/10.1016/j.optcom.2018.10.017

\footnotetext{
Hawliau Cyffredinol / General rights

Copyright and moral rights for the publications made accessible in the public portal are retained by the authors and/or other copyright owners and it is a condition of accessing publications that users recognise and abide by the legal requirements associated with these rights.

- Users may download and print one copy of any publication from the public portal for the purpose of private study or research.

- You may not further distribute the material or use it for any profit-making activity or commercial gain

- You may freely distribute the URL identifying the publication in the public portal ?
}

Take down policy

If you believe that this document breaches copyright please contact us providing details, and we will remove access to the work immediately and investigate your claim. 


\title{
Subcarrier Index-Power Modulated Optical OFDM with Dual
}

\section{Superposition Multiplexing for PON Systems}

\author{
F. Halabi ${ }^{1, *}$, L. Chen ${ }^{2}$, R. P. Giddings ${ }^{1}$, A. Hamié ${ }^{3}$, Y. Dumas ${ }^{4}$, P. Freyssinet ${ }^{4}$, C. Aupetit- \\ Berthelemot $^{5}$ and J. M. Tang ${ }^{1}$ \\ ${ }^{1}$ School of Electronic Engineering, Bangor University, Bangor, LL57 IUT, UK \\ ${ }^{2}$ College of Electronics and Information Engineering, Shanghai University of Electric Power, Shanghai, \\ 200090, China \\ ${ }^{3}$ CRITC Lab, Arts, Sciences and Technology University in Lebanon University, Beirut 11022801, Lebanon \\ ${ }^{4}$ Engineering School ENSIL-ENSCI, University of Limoges, France \\ ${ }^{5}$ XLIM Laboratory, UMR CNRS 7252, University of Limoges, France \\ *Corresponding Author: F. Halabi (eep604@bangor.ac.uk)
}

\begin{abstract}
:
A signal transmission technique termed subcarrier index-power modulated optical orthogonal frequency division multiplexing with dual superposition multiplexing (SIPMOOFDM-DSPM) is proposed and investigated, for the first time, for IMDD PON transmission systems. Compared to previously published similar transmission techniques, SIPM-OOFDM-DSPM not only enables more information bits to be conveyed in the extra information-carrying dimension but also improves the power usage efficiency, performance flexibility and adaptability without increasing DSP and hardware complexity. Detailed numerical simulations of the proposed technique are undertaken to identify optimum transceiver parameters, based on which its performance characteristics are explored. It is shown that over the aforementioned transmission systems, SIPM-OOFDM-DSPM considerably enhances the signal transmission capacity and simultaneously decreases the
\end{abstract}


power penalty, in comparison with other subcarrier-index power-based OOFDM techniques encoded using similar signal modulation formats.

Key words: Optical orthogonal frequency division multiplexing, signal modulation and passive optical networks.

\section{Introduction}

The fifth generation $(5 \mathrm{G})$ of mobile communications are targeted to offer significantly increased signal transmission capacities, massive machine-type communications and ultrareliable low-latency real-time services. To implement 5G networks, a large number of unprecedented technical challenges must be addressed across all layers. In terms of costeffectively realizing $5 \mathrm{G}$ fronthaul links that interconnect multiple cell-site-located remote radio heads (RRHs) with a centralized baseband unit located in a protected location, passive optical networks (PONs) are considered worldwide as one of the most important candidates. As the PON-based fronthaul links should be capable of providing flexible and elastic performance characteristics and dynamic reconfigurablity, highly adaptive signal transmission techniques are thus vital to ensure that the link performance is always optimised regardless of the dynamically changing network connection status. Furthermore, it is also widely envisaged that the initial stage of $5 \mathrm{G}$ should have sufficient transparency to $4 \mathrm{G}$. As such, orthogonal frequency division multiplexing (OFDM) is still a promising signal modulation technique for $5 \mathrm{G}$ because of its unique features including, for example, high spectral efficiency, excellent performance adaptability and cost-effectiveness [1].

To further improve the OFDM transmission performance characteristics to satisfy the aforementioned $5 \mathrm{G}$ requirements, numerous OFDM variants have been proposed recently, which can be divided into three main categories: Category 1 - joint subcarrier and symbol encoding-based OFDM such as set-partitioned OFDM (SP-OFDM) [2], which enhances the 
bandwidth granularity for subcarriers suffering low optical signal-to-noise ratios (OSNRs); Category 2 - OFDM signal multiplexing such as wavelength division multiplexing (WDM) and space division multiplexing (SDM) [3],[4], which improve the signal transmission capacity; and Category 3 - extra information-carrying dimension-based OFDM such as index modulated OFDM (IM-OFDM) [5], which increases the transmission performance flexibility and its susceptibility to low signal to noise ratios (SNRs). In this category, dual-mode OFDM (DM-OFDM) [6] and dual-mode-aided OFDM with constellation power allocation (DMOFDM-CPA) [7] have also been proposed recently for wireless networks. More recently, a survey on the IM technique has been published in [8], where its advantages are summarised in terms of energy efficiency, hardware complexity, system flexibility and performance robustness against inter-channel interference (ICI). Nevertheless, for practical cost-sensitive high-speed 5G optical fronthaul application scenarios, the Category 1 and Category 2 techniques suffer significantly high digital signal processing (DSP) complexity and transceiver hardware complexity respectively. Whilst for the Category 3 techniques, the achievable signal transmission capacity and spectral efficiency are halved compared to conventional OFDM, because almost half of the subcarriers are deactivated.

As a combination of the aforementioned three OFDM categories, a DSP-based signal transmission technique called subcarrier index-power modulated optical OFDM (SIPMOOFDM) has been published in [9], where the combined subcarrier index and subcarrier power acts as an extra information-bearing dimension for carrying additional information bits. In SIPM-OOFDM, subcarriers of high and low powers assigned according to an incoming data sequence are encoded with 8-PSK and QPSK, respectively. To further increase the SIPM-OOFDM signal transmission capacity by more effectively utilizing high power subcarriers, a further improved variant of SIPM-OOFDM termed SIPM-OOFDM with superposition multiplexing (SIPM-OOFDM-SPM) has also been proposed and investigated 
[10]. In SIPM-OOFDM-SPM, the low power subcarriers are still encoded using QPSK, whilst the high power subcarriers use superposition multiplexing (SPM) to passively add two different signal modulation format (for say, 8-PSK and QPSK)-encoded complex numbers, and the resulting sum is then assigned to the subcarriers. Therefore, compared with SIPMOOFDM, the effective usage of high subcarrier powers results in a $28.6 \%$ increase in signal transmission capacity but without increasing the DSP and transceiver hardware complexity. Following a similar technically logical path, it is easy to raise an open question as to whether SPM can be applied on both low and high power subcarriers.

Here it is worth highlighting the fact that in IM, SIPM and SIPM-SPM, various types of energy-free information-bearing dimensions are introduced to convey extra information bits in order to further improve the signal transmission capacity, energy consumption efficiency and system flexibility. However, in IM, only a fraction of certain indexed resource entities are activated for data transmission, whilst the remaining entities are deactivated [8]. In contrast, SIPM and SIPM-SPM always keep the indexed resource entities active, thus leading to the maximized information transmission potential associated with both the newly introduced energy-free information-bearing dimension and the conventional informationbearing dimension.

The thrust of this paper is to address the open question for PON-based fronthaul systems based on intensity modulation and direct detection (IMDD). We propose, for the first time, a new variant of SIPM-OOFDM-SPM, termed SIPM-OOFDM with dual superposition multiplexing (SIPM-OOFDM-DSPM). Compared with SIPM-OOFDM-SPM, the SIPMOOFDM-DSPM encoding procedure on high power subcarriers remain unchanged, whereas SPM is also applied on low power subcarriers by assigning the sum of BPSK- and QPSKencoded complex numbers. Therefore, compared with SIPM-OOFDM-SPM using similar 
signal modulation formats, the proposed technique enables an additional $11 \%$ increase in signal transmission capacity with the transceiver DSP/hardware complexity still preserved.

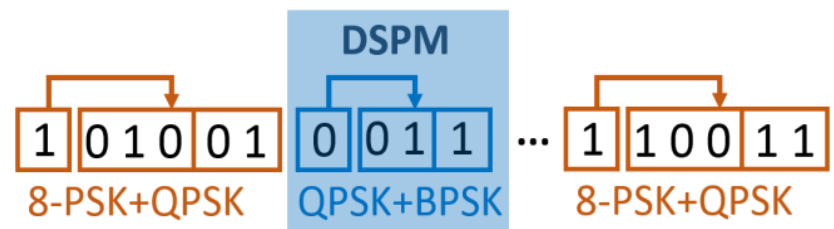

Fig. 1. SIPM-OOFDM-DSPM data-encoding process in the transmitter.

\section{SIPM-OOFDM-DSPM Transceiver Design}

In SIPM-OOFDM-DSPM, the encoding process is similar to SIPM-OOFDM-SPM, where for an incoming PRBS, when a " 1 "' bit is encountered, the corresponding subcarrier is set at a high power level, as illustrated in Fig.1, and the following 5 bits from the PRBS are truncated, of which 3 are encoded using 8-PSK and the remaining 2 bits are encoded using QPSK. Afterwards, these two 8-PSK- and QPSK-encoded complex numbers are added together. This process refers to as the SPM operation. On the other hand, when a " 0 " bit is encountered, the corresponding subcarrier is set at a low power level and the following 3 bits from the sequence are truncated, of which 2 bits are encoded using QPSK, and the remaining

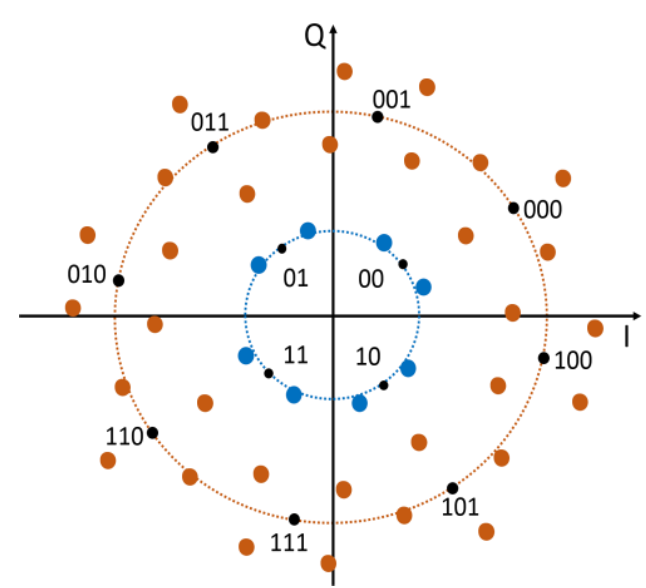

(a)

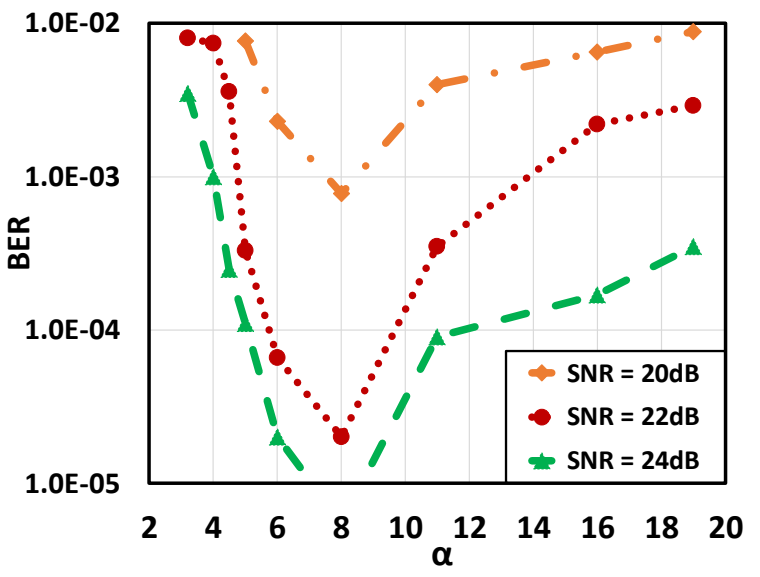

(b)

Fig. 2. (a) Ideal SIPM-OOFDM-DSPM constellation. (b) Overall channel BER versus $\alpha$ over AWGN channels with the SNR values varying from $20 \mathrm{~dB}$ to $24 \mathrm{~dB}$. 
bit is encoded using BPSK. The SPM operation is then performed again by adding these two QPSK- and BPSK- encoded complex numbers together, the resulting sum is subsequently assigned to the low power subcarrier, as illustrated in Fig.1. The ideal SIPM-OOFDM-DSPM constellation is presented in Fig.2(a) in which, for high (low) power subcarriers encoded using 8-PSK- (QPSK) and QPSK (BPSK), DSPM produces four (two) information-carrying satellite constellation points surrounding each virtual 8-PSK (QPSK) point. This gives rise to a total of 32 (8) information-carrying satellite constellation points for high (low) power subcarriers. This indicates that SIPM-OOFDM-DSPM uses low signal modulation formats including 8-PSK, QPSK and BPSK to achieve a signal transmission capacity identical to SIPM-OOFDM encoded using higher signal modulation formats such as 32-PSK and 8-PSK. It is worth mentioning that SIPM-OOFDM-DSPM can be implemented using any signal modulation formats with circular constellations.

It should be noted that since the QPSK modulation format is used on two subcarrier power levels, the optimum $34^{\circ}$ QPSK (BPSK) initial phase setting with respect to 8-PSK (QPSK) reported in [10] is still applicable in SIPM-OOFDM-DSPM for all subcarriers. Moreover, as illustrated in Fig.2(a), a phase rotation of $\pm 90^{\circ}$ should be applied to the BPSK constellation points which are superposed with either the ' 01 ' or ' 10 ' encoded-QPSK point in order to maximize the difference between two subcarrier power levels. As a result, the overall constellation for low power level subcarriers is very similar to 8-PSK, but this offers additional transmission performance advantages, as discussed in Section 3.

To optimize the SIPM-OOFDM-DSPM transceiver parameters, a parameter $\alpha$ is introduced, which represents the ratio between high subcarrier powers and low subcarrier powers. For a fixed total electrical signal power, Fig.2 (b) is plotted to investigate the $\alpha$ impact on the system bit error rate (BER) performance. In simulating this figure, additive white Gaussian noise (AWGN) channels are considered with three SNR values varying from 
$20 \mathrm{~dB}$ to $24 \mathrm{~dB}$. It is shown in Fig.2(b) that the lowest BER is obtainable when $\alpha$ is approximately 8 , and that the optimum $\alpha$ value is SNR-independent. For $\alpha$ values lower than 8 , the BER rises sharply, this results from the fast decrease in the subcarrier power difference between the high and low power subcarriers. Whereas when $\alpha$ exceeds 8 , a relatively slow rise in BER is observed due to the reduction in the minimum Euclidean distance of the 8point constellation taken on the low power subcarriers.

To decode the received signal in the receiver, the subcarrier power detection and threshold decision DSP functions detailed in [9],[10] are still employable for this technique. These DSP functions that are located between the fast Fourier transform (FFT) and channel estimation and equalization, first calculate the optimum power threshold for each individual subcarrier, by making use of a training sequence that is periodically inserted into the user data sequence in the transmitter. The subcarrier power threshold, $P_{\text {threshold }}$, is defined as

$$
P_{\text {threshold }}=\frac{\left(P_{8-P S K+Q P S K}\right)+\left(P_{Q P S K+B P S K}\right)}{2}
$$

where $P_{8-P S K+Q P S K}\left(P_{Q P S K+B P S K}\right)$ is the lowest (highest) subcarrier power of the high (low) power subcarriers. In the SIPM-OOFDM-DSPM decoder, the decoding approach used in

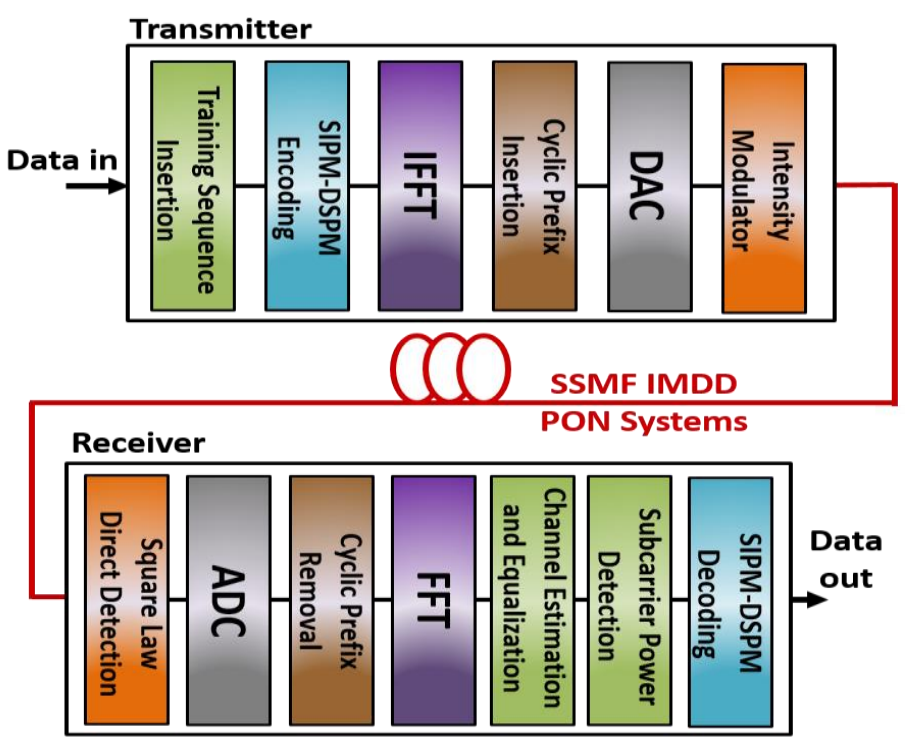

Fig. 3. Schematic illustration of the SIPM-OOFDM-DSPM transceiver architecture and the considered IMDD PON system. 
SIPM-OOFDM-SPM [10] is also applicable in this paper. In this approach, in order to recover the information carried by each high (low) power subcarrier, 32 (8) comparisons between the received complex value $C_{R}^{H}\left(C_{R}^{L}\right)$ and all the possible 32 (8) ideal complex values $C_{I i}^{H}(i=1,2, \ldots, 32)\left(C_{I j}^{L}(j=1,2, \ldots, 8)\right)$ are made. The received complex value which corresponds to the minimum of $\left|C_{R}^{H}-C_{I i}^{H}\right|^{2}\left(\left|C_{R}^{L}-C_{I j}^{L}\right|^{2}\right)$ is used to recover the information conveyed by the high (low) power subcarrier [10].

The SIPM-OOFDM-DSPM transceiver architecture and the IMDD PON transmission system considered in this paper are illustrated in Fig. 3. As seen in this figure, major DSP functions involved in the SIPM-OOFDM-DSPM transmitter are almost identical to those employed in SIPM-OOFDM-SPM [10]. Based on the transceiver architecture and the abovediscussed SIPM-OOFDM-DSPM transceiver design principle, it is easy to understand the following unique features associated with the proposed SIPM-OOFDM-DSPM technique. These features are summarized below:

- High signal transmission capacity enabled by low signal modulation formats;

- Enhanced transceiver flexibility because DSP logic resources remain almost constant for various transmission techniques including SIPM-OOFDM, SIPMOOFDM-SPM and SIPM-OOFDM-DSPM;

- Improved performance adaptability. For different transmission system requirements, simple DSP modifications result in dynamic variations in system transmission performance characteristics (signal bit rate and system power budget) to ensure that the optimum system performance is always delivered regardless of traffic/network status. 
- Low DSP and hardware transceiver complexity. For a specific transceiver architecture, an increase in signal transmission capacity does not require any significant increase in DSP complexity. In terms of hardware, the transceiver design identical to SIPM-OOFDM-SPM [10] is still applicable here. On the other hand, in terms of DSP complexity, the same simple DSP functions used in the SIPMOOFDM-SPM transmitter and receiver are also used in this technique.

\section{SIPM-OOFDM-DSPM Transmission Performance}

Based on the SIPM-OOFDM-DSPM transceiver architecture shown in Fig.3, the optimum parameters identified in Section 2, and the operation parameters listed in Table 1, the transmission performance of the proposed technique is investigated in this section. Throughout this paper, the optimum clipping ratio, quantization bits and DAC/ADC sampling

Table 1 Transceiver and Transmission System Parameters

\begin{tabular}{|c|c|c|}
\hline Parameter & Value & Unit \\
\hline Total number of IFFT/FFT points & \multicolumn{2}{|c|}{64} \\
\hline Data-carrying subcarriers & \multicolumn{2}{|c|}{31} \\
\hline Modulation format & BPSK or QPSK or 8-PSK \\
\hline Cyclic prefix & \multicolumn{2}{|c|}{$25 \%$} \\
\hline PRBS data sequence length & 400,000 & bits \\
\hline DAC \& ADC sample rate & 12.5 & GS/s \\
\hline DAC \& ADC bit resolution & 9 & bits \\
\hline Clipping ratio & 12 & $\mathrm{~dB}$ \\
\hline Modulator and PIN bandwidths & 12.5 & $\mathrm{GHz}$ \\
\hline PIN detector sensitivity & -19 & $\mathrm{dBm}$ \\
\hline PIN responsivity & 0.8 & $\mathrm{~A} / \mathrm{W}$ \\
\hline SSMF dispersion parameter at $1550 \mathrm{~nm}$ & 16 & $\mathrm{ps} /(\mathrm{nm} . \mathrm{km})$ \\
\hline SSMF dispersion slope at $1550 \mathrm{~nm}$ & 0.07 & $\mathrm{ps} / \mathrm{nm} / \mathrm{nm} / \mathrm{km}$ \\
\hline Linear fiber attenuation & 0.2 & $\mathrm{~dB} / \mathrm{km}$ \\
\hline Kerr coefficient & $2.35 \times 10^{-20}$ & $\mathrm{~m} / \mathrm{W}$ \\
\hline
\end{tabular}

${ }^{*}$ Corresponding to $10 \mathrm{~Gb} / \mathrm{s}$ non-return-to-zero data at a BER of $1.0 \times 10^{-9}$ 
Table 2 Signal Transmission Bit Rate Comparisons

\begin{tabular}{|c|c|}
\hline Modulation Format & Signal Bit Rate $(\mathrm{Gb} / \mathrm{s})$ \\
\hline SIPM-OOFDM-SPM & 26.71 \\
\hline SIPM-OOFDM-DSPM & 29.73 \\
\hline SIPM-OOFDM (32-PSK/8-PSK) & 29.73 \\
\hline
\end{tabular}

rates are fixed at $12 \mathrm{~dB}, 9$ bits and 12.5GS/s, respectively [9],[10]. As shown in Fig.4(a), compared to SIPM-OOFDM-SPM, SIPM-OOFDM-DSPM has an almost identical peak-toaverage power ratio (PAPR) cumulative distribution function (CDF) curve. As a direct result, the same optimum clipping ratios between these two techniques are observed in Fig.4(b). It can be seen in Fig.4(b) that the optimum clipping ratios of $12 \mathrm{~dB}$ give rise to minimum BERs for both techniques. In calculating Fig.4(b), an AWGN channels with a SNR value of $22 \mathrm{~dB}$ is considered.

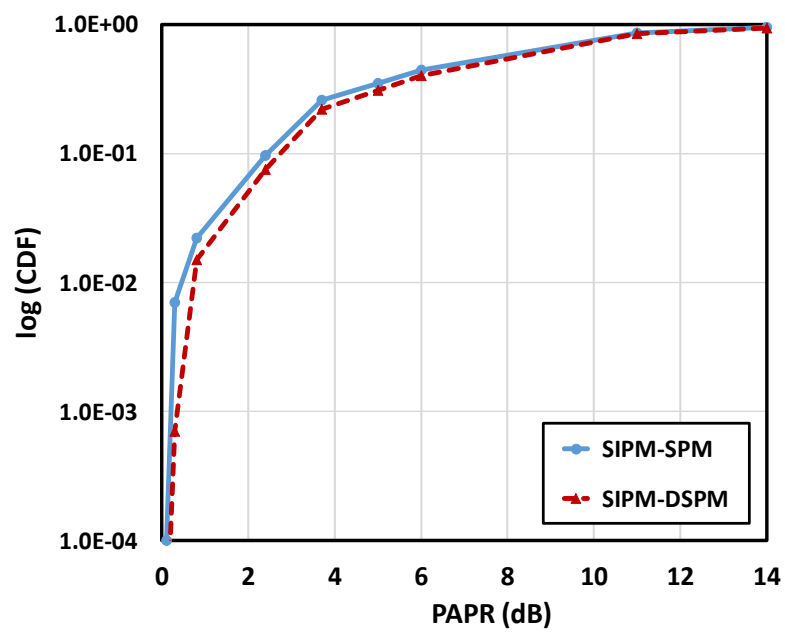

(a)

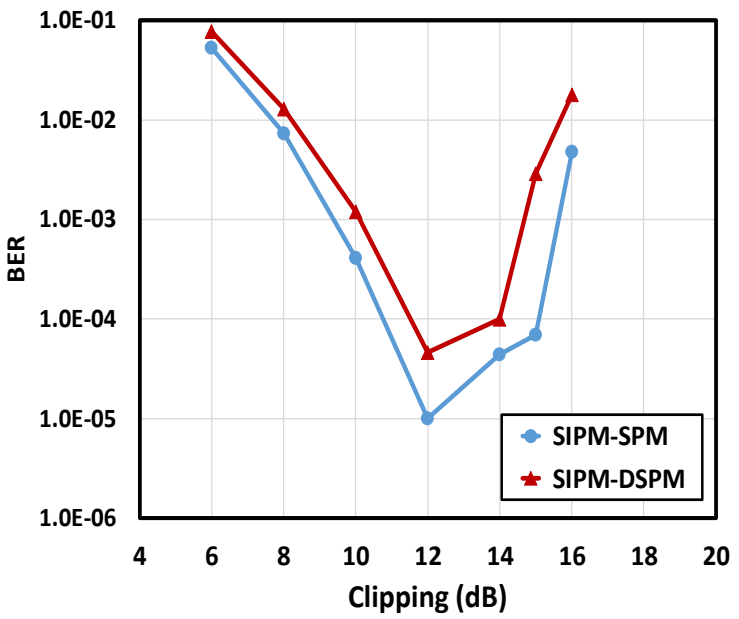

(b)

Fig. 4. (a) Cumulative distribution functions for both SIPM-OOFDM-SPM and SIPM-OOFDM-DSPM. (b) BER performance versus clipping ratio over AWGN channels with a SNR value fixed at $22 \mathrm{~dB}$.

By making use of the signal bit rate calculation formula reported in [10], the SIPMOOFDM-DSPM signal bit rate can be easily computed and compared with other transmission techniques of similar nature, as summarized in Table 2. In this table, it is shown that the proposed technique gives rise to a signal bit rate of $29.73 \mathrm{~Gb} / \mathrm{s}$, which outperforms (8- 


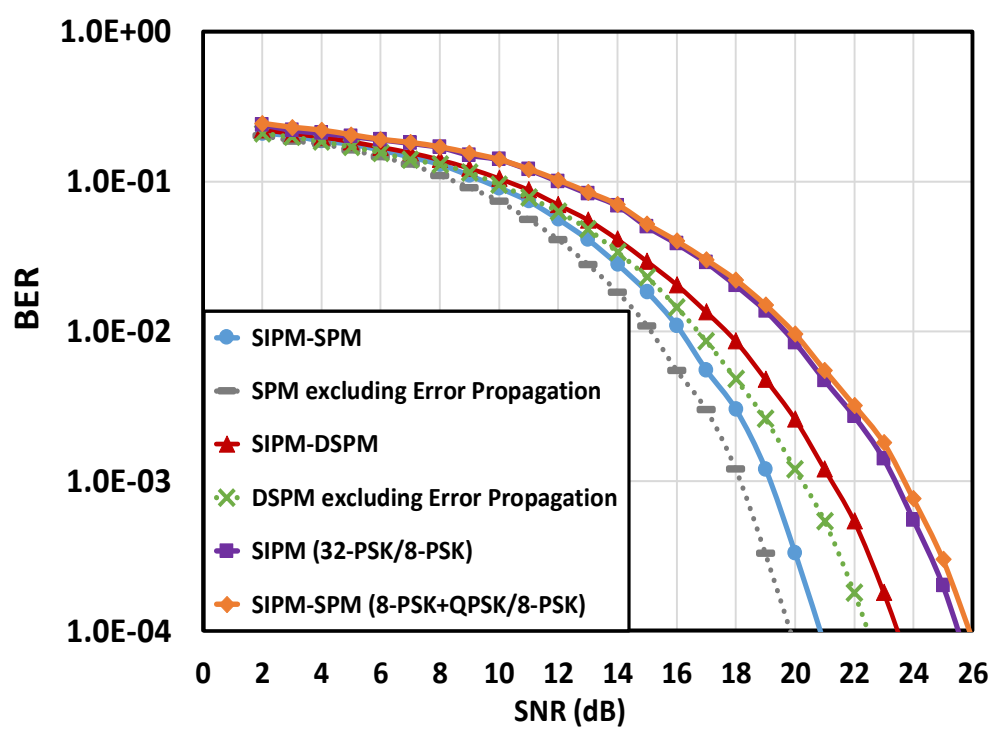

Fig. 5. BER performance versus signal SNR over AWGN channels for various transmission techniques.

PSK+QPSK)/QPSK-encoded SIPM-OOFDM-SPM by 11\%. Table 2 also indicates that SIPM-OOFDM-DSPM supports the same signal bit rate as the 32-PSK/8-PSK-encoded SIPM-OOFDM. The impacts of DSPM on minimum required electrical SNR over AWGN channels are presented in Fig. 5. It is shown that the 29.73Gb/s SIPM-OOFDM-DSPM signal introduces an SNR penalty of approximately $1.9 \mathrm{~dB}$ at a BER of $1.0 \times 10^{-3}$ in comparison with the $26.71 \mathrm{~Gb} / \mathrm{s} \quad$ (8-PSK+QPSK)/QPSK-encoded SIPM-OOFDM-SPM signal. More importantly, the proposed technique offers almost $2.5 \mathrm{~dB}$ gain at a BER of $1.0 \times 10^{-3}$ when compared with both the $29.73 \mathrm{~Gb} / \mathrm{s}$ 32-PSK/8-PSK-encoded SIPM-OOFDM and (8PSK+QPSK)/8-PSK-encoded SIPM-OOFDM-SPM signals. Such SNR gains confirm that the DSPM operation offers a considerable performance improvement compared with the use of only 8-PSK in low power subcarriers.

The error propagation-free approach reported in [9],[10] is also applicable in this technique. In this approach, the signal modulation formats taken on each individual subcarrier are compared between the transmitter and the receiver. A difference in signal modulation formats indicates the occurrence of a subcarrier power detection error, the corresponding error bits conveyed by the subcarrier are removed, and a corresponding number of random 
bits is added (removed) when a lower (higher) signal modulation format is detected in the receiver compared to the transmitter [9]. As shown in Fig.5, when applying such an approach in SIPM-OOFDM-DSPM, the same impact resulting from excluding error propagation in SIPM-OOFDM-SPM [10] is also observed here where an SNR gain of approximately 1.0dB is achieved. In the remaining parts of the paper, error propagation is excluded in both SIPMOOFDM-SPM and SIPM-OOFDM-DSPM.

Based on the fiber transmission parameters identical to those reported in [10], a VPIphotonics simulation model is used here to investigate the $29.73 \mathrm{~Gb} / \mathrm{s}$ SIPM-OOFDMDSPM transmission performances over 25km SSMF IMDD PON systems.

In this paper, an ideal intensity modulator is adopted which produces an optical field output signal, $S_{O}(t)$, having a waveform governed by

$$
S_{O}(t)=\sqrt{S e(t)}
$$

where $S e(t)$ is the electrical driving current of the SIPM-OOFDM-DSPM signal with an optimum dc bias current being added. In the receiver, a PIN with a receiver sensitivity of $-19 \mathrm{dBm}$ is also employed with optical launch powers fixed at $5 \mathrm{dBm}$. Both shot noise and

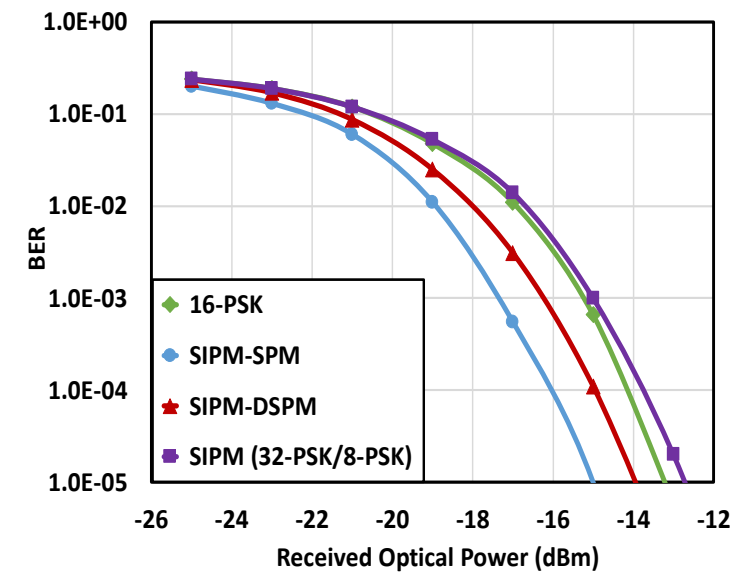

(a)

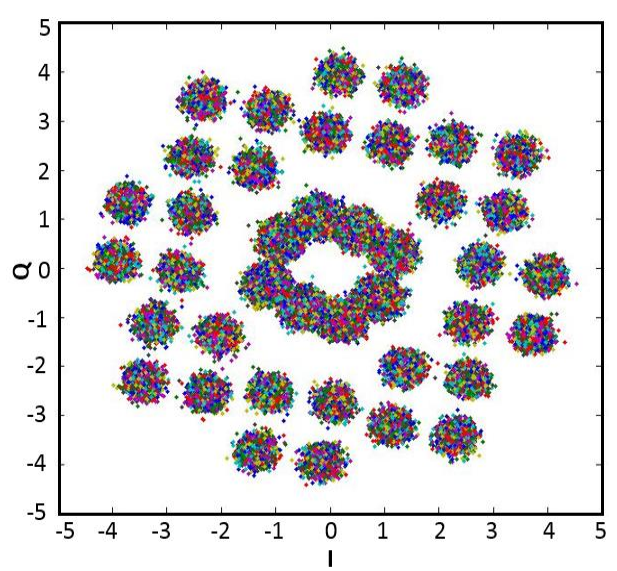

(b)

Fig. 6. (a) Overall channel BER versus received optical power for various transmission techniques. (b) SIPM-OOFDM-DSPM constellation. 
thermal noise are considered, which are simulated utilizing the procedures similar to those presented in [11].

In Fig.6(a), the BER performance comparison of the 29.73Gb/s SIPM-OOFDM-DSPM signal with both the $26.71 \mathrm{~Gb} / \mathrm{s}$ (8-PSK+QPSK)/QPSK-encoded SIPM-OOFDM-SPM signal and the $29.73 \mathrm{~Gb} / \mathrm{s} 32-\mathrm{PSK} / 8$-PSK-encoded SIPM-OOFDM signal shows that there exists a $0.9 \mathrm{~dB}$ power penalty and a $1.8 \mathrm{~dB}$ received optical power gain respectively at a BER of $1.0 \times 10^{-3}$. In addition, when compared with a $23.73 \mathrm{~Gb} / \mathrm{s}$ 16-PSK-encoded conventional OOFDM signal, a $1.7 \mathrm{~dB}$ received optical power gain is achieved at a BER of $1.0 \times 10^{-3}$. Similar to the results presented in [10], our results show that the 29.73Gb/s SIPM-OOFDMDSM signal improves the dispersion tolerance range by almost $75 \mathrm{ps} / \mathrm{nm}$ at a BER of $1.0 \times 10^{-}$ ${ }^{3}$, compared to the $29.73 \mathrm{~Gb} / \mathrm{s}$ (32-PSK/8-PSK) SIPM-OOFDM signal. The physical origin of such an improvement is mainly due to the use of low signal modulation formats in low power subcarriers. As a direct result of the same physical mechanism, our results also show that, for achieving a BER of $1.0 \times 10^{-3}$, the proposed technique can improve the optical launch power dynamic range by $3 \mathrm{~dB}$ compared to the $29.73 \mathrm{~Gb} / \mathrm{s}$ (32-PSK/8-PSK) SIPM-OOFDM-SPM signal. The above discussions indicate that for practical applications, the signal modulation format selection can be adaptive and flexible: for power budget-limited transmission systems, relatively low signal modulation formats are preferred to increase the system power budget, on the other hand, for bandwidth-hungry transmission systems with sufficiently large power budgets, relatively high signal modulation formats are preferred to maximise the signal transmission capacity. 
Table 3 SIPM-OOFDM-DSPM Performance Comparisons

\begin{tabular}{|c|c|c|}
\hline Modulation Format & Signal Bit Rate & Power Penalty $(\mathrm{dB})$ \\
\hline 16-PSK-OOFDM & $25 \%$ Increase & -1.7 \\
\hline SIPM-OOFDM-SPM & $11 \%$ Increase & 0.9 \\
\hline SIPM-OOFDM (32-PSK/8-PSK) & Identical & -1.8 \\
\hline
\end{tabular}

As seen in Table 3, the DSPM-introduced 11\% increase in signal transmission capacity causes only $0.9 \mathrm{~dB}$ changes to the system power budget for a specific BER. This table also shows that, in comparison with the 32-PSK/8-PSK-encoded SIPM-OOFDM technique capable of offering a signal transmission capacity identical to SIPM-OOFDM-DSPM, the proposed technique improves the system power budget by $1.8 \mathrm{~dB}$. Table 3 also shows that, in comparison with 16-PSK-encoded conventional OOFDM, a 25\% increase in signal bit rate as well as a $1.7 \mathrm{~dB}$ gain in system power budget is achieved. Finally, the SIPM-OOFDM-DSPM constellation obtained after equalization at a BER of $1.0 \times 10^{-3}$ is illustrated in Fig.6(b).

\section{Conclusions}

As an improved variant of the previously published SIPM-OOFDM-SPM technique, SIPM-OOFDM-DSPM, has been proposed and investigated, for the first time, for use in SSMF IMDD PON systems for 5G fronthaul links. Optimum key transceiver parameters have been identified numerically, based on which the SIPM-OOFDM-DSPM transmission performance characteristics have been explored. It is shown that, with preserved DSP and hardware complexity, SIPM-OOFDM-DSPM offers an $11 \%$ improvement in signal transmission capacity compared to (8-PSK+QPSK)/QPSK-encoded SIPM-OOFDM-SPM, and $1.8 \mathrm{~dB}$ received optical power gain compared to 32-PSK/8-PSK-encoded SIPM-OOFDMSPM. 


\section{References}

[1] G. Berardinelli et al., "On the potential of OFDM enhancements as 5G waveforms,', Veh. Tech. Conf. (VTC Spring), pp. 1-5, May 2014.

[2] J. Zhao and C. K. Chan, "Adaptively loaded SP-offset-QAM OFDM for IM/DD communication systems,” Opt. Exp., vol. 25, no. 8, pp. 21603-21618, Sep. 2017.

[3] H. Saito et al., "Adaptive bandwidth allocation algorithm for WDM/OFDM-PON-based elastic lambda aggregation network," Opt. Fibre Commun. Conf. (OFC), Th3C.2, pp.1-3, Mar. 2016.

[4] P. Khodashenas et al., "Comparison of spectral and spatial super-channel allocation schemes for SDM networks,” J. Lightw. Technol., vol. 34, no. 11, pp. 2710-2716, Jun. 2016.

[5] S. Dang, J. P. Coon and G. Chen, “Adaptive OFDM with index modulation for two-hop relay-assisted networks,' IEEE Trans. on Wireless Commun., vol. 17, no. 3, pp. 1923-1936, Mar. 2018.

[6] T. Mao, Z. Wang, Q. Wang, S. Chen, and L. Hanzo, "Dual-mode index modulation aided OFDM," IEEE Access, vol. 5, pp. 50-60, 2017.

[7] X. Zhang, H. Bie, Q. Ye, C. Lei, X. Tang, "Dual-Mode Index Modulation Aided OFDM With Constellation Power Allocation and Low-Complexity Detector Design", IEEE Access, vol. 5, pp. 23871-23880, 2017.

[8] T. Mao, Q. Wang, Z. Wang, S. Chen, "Novel Index Modulation Techniques A Survey", IEEE Communications Surveys \& Tutorials, DOI: 10.1109/COMST.2018.2858567, 2018.

[9] F. Halabi et al., "Subcarrier index-power modulated optical OFDM and its performance in IMDD PON systems,” J. Lightw. Technol., vol. 34, no. 9, pp. 2228-2234, May 2016. 
[10] L. Chen et al., "Subcarrier index-power modulated optical OFDM with superposition multiplexing for IMDD transmission systems," J. Lightw. Technol., vol. 34, no. 9, pp. 22282234, Oct. 2016.

[11] G. P. Agrawal, Fibre-Optic Communication Systems, 2nd ed. Hoboken, NJ, USA: Wiley, 1997. 